\title{
Decentralized string-stability analysis for heterogeneous cascades subject to load-matching requirements
}

\author{
Laven Soltanian · Michael Cantoni
}

Received: date / Accepted: date

\begin{abstract}
A heterogeneous cascade of stable linear time-invariant subsystems is studied in terms of the spatial and temporal propagation of boundary conditions. The particular context requires constant spatial boundary conditions to be asymptotically matched by the interconnection signals along the string (e.g., to match supply to demand in steady state). Furthermore, the transient response associated with a step change in the spatial boundary condition must remain bounded across space in a string-length independent fashion. With this in mind, an infinite cascade abstraction is considered. A corresponding decentralized string-stability certificate for the desired behaviour is established in terms of the subsystem $H_{\infty}$ norms, via Lyapunovtype analysis of a two-dimensional model in Roesser form. Verification of the certificate implies uniformly bounded interconnection signals in response to the following system inputs: $(i)$ a square-summable (across space) sequence of initial conditions; and (ii) a uniformly-bounded (across time) finite-energy input applied as the spatial boundary condition (e.g., finite duration on-off pulse). The decentralized nature of the certificate facilitates subsystem-by-subsystem design of local controllers that achieve string-stable behaviour overall. This application of the analysis is explored within the context of a scalable approach to the design of distributed distant-downstream controllers for the sections of an automated irrigation channel.
\end{abstract}

\section{Introduction}

Aspects of temporal and spatial stability are investigated for infinite cascades of stable linear time-invariant (LTI) systems. Specifically, interconnections of the kind shown

L. Soltanian

Department of Electrical and Electronic Engineering, University of Melbourne, VIC 3010, Australia

E-mail: 1.soltanian@student.unimelb.edu.au

Present address: Rubicon Water, Pty. Ltd., Hawthorn East, VIC 3123, Australia

M. Cantoni

Department of Electrical and Electronic Engineering, University of Melbourne, Victoria 3010, Australia

E-mail: cantoni@unimelb.edu.au 
in Figure 1 are studied. Such models arise in various application domains, such as vehicle platoons [16,3,11,6,9,23, 12, 13], air traffic control [28, 1], supply chains [10, 7], and automated irrigation channels [2,26], for example.

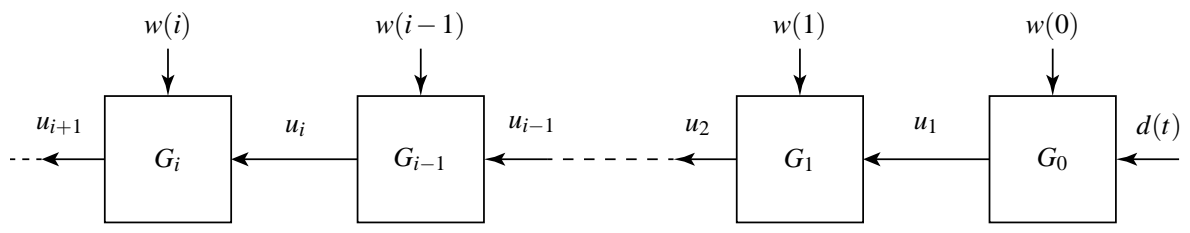

Fig. 1 Cascade of heterogeneous dynamical systems.

In Figure1 the interconnection signal $u_{i+1}$ at the output of subsystem $i \geq 0$ is related to the interconnection signal $u_{i}$ at the corresponding input, according to an LTI dynamic input-output relationship $u_{i+1}=G_{i} u_{i}$. The corresponding spatial boundary condition is $u_{0}=d(t)$, where $d(t)$ is a time-dependent input signal. The constant value $w(i)$ is an input associated with non-zero initialization of a state-space realization for $G_{i}$. As such, the sequence $\{w(0), w(1), \ldots\}$ constitutes a space-dependent temporal boundary condition for a state-space realization of the cascade. An important aspect of such system interconnections is the unidirectional propagation of information in both space, from right to left as shown in Figure 1 and in time.

Many papers in the literature consider the propagation of boundary disturbances and/or initial conditions along a chain or string of dynamical subsystems. Early work in this direction was carried out within the context of optimal error control for a platoon of vehicles [16]. In [3], "stability of a string" is defined in terms of requiring bounded position error fluctuations that also tend to zero in steady state, in response to bounded initial conditions for all vehicles. See [13] for a recent overview of various definitions of string-stability.

In this paper, cascades of the kind described above are studied from the perspective of $L_{2}$-to- $L_{\infty}$ string-stability. That is, in terms of desiring uniformly bounded, across time and space, interconnection signals in response to bounded finite-energy spatial and temporal boundary conditions. Limiting attention to finite-energy boundary conditions is motivated by the finite duration of typical spatial boundary inputs and the finite extent of cascades in practice. The infinite cascade abstraction is nonetheless relevant, as uniformity requirements within such a context translate to time- and space-horizon independent bounds, which is important within the context of long chains.

The main result is a decentralized $L_{2}$-to- $L_{\infty}$ string-stability certificate applicable to heterogeneous cascades, subject to the requirement that constant spatial boundary conditions are asymptotically matched by the interconnection signals along the chain of subsystems. This is motivated by the operation of automated irrigation channels, where a steady-state objective at each flow regulating structure is to match the downstream load, as may also arise in other application domains. The string-stability certificate involves verification of the location independent property that the $H_{\infty}$ norm of the interconnection-signal transfer function of each subsystem be no greater than 
unity. This is more amenable to design and synthesis than impulse-response based certificates, which ensure a related but more stringent string-stability property [26, 25]. The main result extends earlier work reported in [13,14], where the stability of homogeneous platoons of vehicles are studied in a similar fashion, via Lyapunovbased stability analysis (see e.g. [8]) of a two-dimensional (2-D) Roesser model for the cascade, although an analog of the steady-state matching requirement is not considered explicitly therein.

The main result is also distinct from similar conditions in the literature in that the corresponding $H_{\infty}$-norm bound condition is non-strict, as required to accommodate the aforementioned steady-state matching requirement, unlike the strict norm-bound conditions presented in [24,6,5] for various notions of string stability. It is interesting to note that the non-strict certificate developed here is necessary for the different notion of string stability considered in [21]. Indeed, this observation is presented in [21] as a motivation for using bi-directional information exchange in vehicle platoons, as it is not possible to achieve the necessary condition with uni-directional information flow. Bi-directional information exchange would be disadvantages within the context of irrigation channels, and other distribution networks, in view of the typically limited availability of downstream storage. It is also of note that results of the kind considered in [15], which are also applicable to interconnections of heterogeneous subsystems, do not directly yield the uniform $L_{\infty}$ bounds on the system response to boundary conditions, as required here.

The decentralized nature of the string-stability certificate developed in this paper can facilitate the design of cascades on a subsystem-by-subsystem basis. This scalability aspect of the result is explored within the context of so-called distributed distant-downstream control architectures for automated irrigation channels. Within this context, it is desirable from an engineering perspective for each distributed component of the controller to be designed using only the model of the corresponding section of the channel dynamics. This can be advantageous in terms of the tractability of systematic approaches to controller synthesis and system maintainability.

The paper is structured as follows. Section 2 sets some basic notation and preliminary technical results. A 2-D Roesser model is then developed for a heterogeneous cascade in Section 3 . The main $L_{2}$-to- $L_{\infty}$ string-stability analysis results, including the aforementioned decentralized certificate, are presented in Section 4 Section 5 contains an application of the analysis within the context of irrigation channel controlsystem design. Some final remarks are provided in a concluding section.

\section{Notation and Preliminaries}

The symbol $\mathbb{Z}_{+}$denotes the subset $\{i \in \mathbb{Z}: i \geq 0\}$ of the integers $\mathbb{Z}, \mathbb{R}_{+}$denotes the subset $\{t \in \mathbb{R}: t \geq 0\}$ of the real numbers $\mathbb{R}$, and $\mathbb{C}_{-}$denotes the subset $\{z \in \mathbb{C}$ : $\mathfrak{R}(z)<0\}$ of the complex numbers $\mathbb{C}=\{\alpha+j \beta: \alpha, \beta \in \mathbb{R}\}$, where $\mathfrak{R}(z)$ denotes the real part $\alpha$ of $z=\alpha+j \beta$ and $j:=\sqrt{-1}$. The symbol $\mathbb{R}^{n}$ denotes the linear space of column vectors with $n$ real-valued entries. $\mathbb{F}^{p \times m}$ denotes the linear space $p$-row-by$m$-column matrices with entries in $\mathbb{F} \in\{\mathbb{R}, \mathbb{C}\}$. A superscript $*$ denotes the (complex conjugate) transpose of a matrix or column vector considered as an $n \times 1$ matrix. 
Given a vector $x=\left(x_{1}, \ldots, x_{n}\right)^{*} \in \mathbb{R}^{n},|x|_{2}:=\left(\sum_{i=1}^{n} x_{i}^{2}\right)^{\frac{1}{2}}$ and $|x|_{\infty}:=\max \left\{\left|x_{i}\right|:\right.$ $i=1,2, \ldots, n\}$. Note that $|x|_{\infty} \leq|x|_{2}=\sqrt{x^{*} x}$ for all $x \in \mathbb{R}^{n}$. Given $A \in \mathbb{R}^{p \times m}$ with elements $a_{i j} \in \mathbb{R},\|A\|_{2 \rightarrow 2}:=\sup _{x \neq 0}|A x|_{2} /|x|_{2}$ and $\|A\|_{\infty \rightarrow \infty}:=\sup _{x \neq 0}|A x|_{\infty} /|x|_{\infty}=$ $\max _{i} \sum_{j} a_{i j}$. The set of eigenvalues of $A$ is denoted by $\sigma(A)=\{\lambda \in \mathbb{C}: A x=\lambda x, x \neq$ $0\}$. Clearly, $\lambda \in \sigma(A) \Leftrightarrow-\lambda \in \sigma(-A)$ and $\lambda \in \sigma(A) \Leftrightarrow(1+\lambda) \in \sigma(I+A)$, where $I$ denotes the identity matrix.

A symmetric matrix $P=P^{*} \in \mathbb{R}^{n \times n}$ has eigenvalues that are real and $\lambda_{\max }(P)$ (resp. $\left.\lambda_{\min }(P)\right)$ denotes the maximum (resp. minimum) eigenvalue. It is said to be positive definite (resp. semi-definite) if $x^{*} P x \geq c x^{*} x$ (resp. $x^{*} P x \geq 0$ ) for all $x \in \mathbb{R}^{n}$ and some $c>0$, which is denoted by $P>0$ (resp. $P \geq 0$ ). Also, $P<0 \Leftrightarrow-P>0$ and $P \leq 0 \Leftrightarrow-P \geq 0$. Note that $P>0 \Leftrightarrow \lambda_{\min }(P)>0$ and $P<0 \Leftrightarrow \lambda_{\max }(P)<0$. Given $P>0$, there exists a unique matrix $0<P^{\frac{1}{2}}=\left(P^{\frac{1}{2}}\right)^{*} \in \mathbb{R}^{n \times n}$ such that $P=P^{\frac{1}{2}} P^{\frac{1}{2}}$.

$L_{2}^{n}$ denotes the space of functions $x: \mathbb{R}_{+} \rightarrow \mathbb{R}^{n}$ with $\|x\|_{2}:=\left(\int_{0}^{\infty}|x(t)|_{2}^{2} d t\right)^{\frac{1}{2}}<\infty$. The space of functions $x: \mathbb{R}_{+} \rightarrow \mathbb{R}^{n}$ such that $\|x\|_{\infty}:=\sup _{t}|x(t)|_{\infty}<\infty$ is denoted by $L_{\infty}^{n}$. Similarly, $\ell_{2}^{n}$ and $\ell_{\infty}^{n}$ denote the subspaces of sequences $x: \mathbb{Z}_{+} \rightarrow \mathbb{R}^{n}$ such that $\|x\|_{2}:=\left(\sum_{i=0}^{\infty}|x(i)|_{2}^{2}\right)^{\frac{1}{2}}<\infty$ and $\|x\|_{\infty}:=\sup _{i}|x(i)|_{\infty}<\infty$, respectively. The dimension $n$ of the function value is often suppressed for convenience.

The following technical lemma, a version of which can be found in [13] for example, is used subsequently in Lyapunov type analysis of a 2-D Roesser model for heterogeneous cascades.

Lemma 1 Let $V_{t}, V_{s}: \mathbb{R}_{+} \times \mathbb{Z}_{+} \rightarrow \mathbb{R}$. If $V_{t}(t, i) \geq 0, V_{s}(t, i) \geq 0$ and

$$
\Delta(t, i):=\frac{\partial V_{t}}{\partial t}(t, i)+V_{s}(t, i+1)-V_{s}(t, i) \leq 0
$$

for all $(t, i) \in \mathbb{R}_{+} \times \mathbb{Z}_{+}$, then

$$
\begin{aligned}
& \sum_{k=0}^{i} V_{t}(t, k) \leq \sum_{k=0}^{i} V_{t}(0, k)+\int_{0}^{t} V_{s}(\tau, 0) d \tau \text { and } \\
& \int_{0}^{t} V_{s}(\tau, i) d \tau \leq \int_{0}^{t} V_{s}(\tau, 0) d \tau+\sum_{k=0}^{i} V_{t}(0, k)
\end{aligned}
$$

for all $(t, i) \in \mathbb{R}_{+} \times \mathbb{Z}_{+}$.

Proof Since $\Delta(t, i) \leq 0$ for all $(t, i) \in \mathbb{R}_{+}$,

$$
0 \geq \sum_{k=0}^{i} \int_{0}^{t} \Delta(\tau, k) d \tau=\sum_{k=0}^{i}\left(V_{t}(t, k)-V_{t}(0, k)\right)+\int_{0}^{t}\left(V_{s}(\tau, i)-V_{s}(\tau, 0)\right) d \tau,
$$

Therefore,

$$
\sum_{k=0}^{i} V_{t}(t, k)+\int_{0}^{t} V_{s}(\tau, i) d \tau \leq \sum_{k=0}^{i} V_{t}(0, k)+\int_{0}^{t} V_{s}(\tau, 0) d \tau
$$

whereby (1) and (2) hold because $V_{t}(t, i) \geq 0$ and $V_{s}(t, i) \geq 0$. 
The following non-strict version of the so-called Bounded Real Lemma plays a role in subsequently establishing a decentralized string-stability certificate for heterogeneous cascades of stable LTI subsystems with rational transfer functions.

Lemma 2 Let $G(s)=C(s I-A)^{-1} B$ be a state-space realization of a strictly-proper matrix transfer function, with $A \in \mathbb{R}^{n \times n}$ Hurwitz (i.e. $\sigma(A) \subset \mathbb{C}_{-}$). The following are equivalent:

(i) $\|G\|_{\infty}:=\sup _{\mathfrak{R}(s)>0}\|G(s)\|_{2 \rightarrow 2}=\sup _{\omega \in \mathbb{R}}\|G(j \omega)\|_{2 \rightarrow 2} \leq 1$;

(ii) there exists a unique real matrix $X=X^{*} \geq 0$ such that

$$
A^{*} X+X A+X B B^{*} X+C^{*} C=0
$$

and $\sigma\left(A+B B^{*} X\right) \subset \mathbb{C}_{-} \cup j \mathbb{R}$.

Proof $\|G\|_{\infty} \leq 1$ is equivalent to

$$
\begin{aligned}
\phi(j \omega): & =I-G^{*}(j \omega) G(j \omega) \\
& =\left[B^{*}\left(-j \omega I-A^{*}\right)^{-1} I\right]\left[\begin{array}{cr}
-C^{*} C & 0 \\
0 & I
\end{array}\right]\left[\begin{array}{c}
(j \omega I-A)^{-1} B \\
I
\end{array}\right] \geq 0 .
\end{aligned}
$$

Applying [31, Lemma 13.17], $\phi(j \omega) \geq 0$ for all $\omega \geq 0$ is equivalent to the existence of a unique real $Y=Y^{*} \leq 0$ such that

$$
A^{*} Y+Y A-Y B B^{*} Y-C^{*} C=0
$$

and $\sigma\left(A-B B^{*} Y\right) \in \mathbb{C}_{-} \cup j \mathbb{R}$. By taking $X=-Y$, this is in turn equivalent to the existence of $X \geq 0$ such that (3) holds with $\sigma\left(A+B B^{*} X\right) \in \mathbb{C}_{-} \cup j \mathbb{R}$.

In the case that additional hypotheses on the state-space realization of $G$ hold, an eigenvalue upper bound for the solution of (3) follows as summarised below. This bound also plays a role in subsequent analysis.

Lemma 3 Let $G(s)=B(s I-A)^{-1} C$, with $A \in \mathbb{R}^{n \times n}$ Hurwitz, $(A, B)$ controllable and $(C, A)$ observable; i.e. $(A, B, C)$ is a minimal realization of $G(\cdot)$. If $\|G\|_{\infty} \leq 1$, then the unique positive semi-definite solution of (3) such that $\sigma\left(A+B B^{*} X\right) \in \mathbb{C}_{-} \cup j \mathbb{R}$ also satisfies $X>0$. Moreover, $\lambda_{\max }(X) \leq 1 / \lambda_{\min }(P)$, where $P=P^{*} \in \mathbb{R}^{n \times n}>0$ is the unique solution of

$$
A P+P A^{*}+B B^{*}=0 .
$$

Proof Since $\|G\|_{\infty} \leq 1$, there exists a unique $X=X^{*} \geq 0$ such that (3) holds with $\sigma\left(A+B B^{*} X\right) \in \mathbb{C}_{-} \cup j \mathbb{R}$ by Lemma 2 Note that $(C, A)$ observable implies $X>0$. To see this, suppose to the contrary that $\operatorname{ker}(X) \neq\{0\}$ and observe the following: (a) $\operatorname{ker}(X) \subset \operatorname{ker}(C)$, which can be seen by left and right multiplying (3) by $x^{*}$ and $x$, to yield $C x=0$ whenever $x \in \operatorname{ker}(X)$; and (b) $A \operatorname{ker}(X) \subset \operatorname{ker}(X)$, which can be seen by right multiplying (3) by $x \in \operatorname{ker}(X)$ so that using (a) it follows that $X A x=0$ and thus, $A x \in \operatorname{ker}(X)$. In particular, (b) implies there exists $0 \neq x \in \operatorname{ker}(X)$ and $\lambda \in \sigma(A) \subset \mathbb{C}_{-}$ such that $A x=\lambda x$. Moreover, $C x=0$ by (a), which contradicts the hypothesis that $(C, A)$ is observable. As such, $\operatorname{ker}(X)=\{0\}$, whereby $X>0$ as claimed. 
Now, let $Z=X^{-1}>0$. Using (3) yields

$$
A Z+Z A^{*}+B B^{*}+Z C^{*} C Z=0
$$

and

$$
A(Z-P)+(Z-P) A^{*}+Z C^{*} C Z=0,
$$

where $P=P^{*}$ is the unique solution of (5), which satisfies $P>0$ because $A$ is Hurwitz and $(A, B)$ is controllable; see [31, Lemma 3.18(iii)]. Since $C^{*} C \geq 0$, it follows from (7) that $A(Z-P)+(Z-P) A^{*} \leq 0$, whereby [31, Lemma 3.18(ii)] implies $Z-P \geq$ 0 . As such, $0 \leq X^{-1}-P=X^{-\frac{1}{2}}\left(I-X^{\frac{1}{2}} P X^{\frac{1}{2}}\right) X^{-\frac{1}{2}}$, which is equivalent to $0 \leq I-$ $X^{\frac{1}{2}} P X^{\frac{1}{2}}$. In particular, $0 \leq \lambda_{\min }\left(I-X^{\frac{1}{2}} P X^{\frac{1}{2}}\right)=1-\lambda_{\max }\left(X^{\frac{1}{2}} P X^{\frac{1}{2}}\right)=1-\lambda_{\max }(X P)$, where $\lambda_{\max }(X)>0$ or $\lambda_{\max }(P)>0$ can be used to establish the last equality. The inequality $\lambda_{\min }(P) \lambda_{\max }(X) \leq \lambda_{\max }(X P)$, which holds by [27, Theorem 2] as $X>0$ and $P>0$, thus leads to the bound $\lambda_{\max }(X) \leq 1 / \lambda_{\min }(P)$.

Remark 1 In Lemma 3 , if $(A, B, C)$ is also a balanced realization of $G$, so that $P=$ $\operatorname{diag}\left(\sigma_{1}, \cdots, \sigma_{n}\right)$ is a diagonal matrix of the Hankel singular values of $G$ in descending order $\sigma_{1} \geq \cdots \geq \sigma_{n}>0$ and $P A+A^{*} P+C C^{*}=0$, then $\lambda_{\max }(X) \leq 1 / \sigma_{n}$.

\section{A 2-D Roesser Model for Heterogeneous Cascades}

Recall the cascade of heterogeneous LTI systems shown in Figure 1 Let

$$
G_{i}(s)=C(i)(s I-A(i))^{-1} B(i)
$$

be a state-space realization for the transfer function of subsystem $i \in \mathbb{Z}_{+}$. In the frequency domain,

$$
U_{i+1}(s)=G_{i}(s) U_{i}(s)+H_{i}(s) w(i)
$$

for $i \in \mathbb{Z}_{+}$, with $U_{0}(s)=D(s)$ and $H_{i}(s):=C(i)(s I-A(i))^{-1}$, where $U_{i}(s)$ and $D(s)$ denote the Laplace transforms of $u_{i}$ and $d$, respectively. In the time domain,

$$
\begin{aligned}
\dot{x}_{i}(t) & =A(i) x_{i}(t)+B(i) u_{i}(t), \\
u_{i+1}(t) & =C(i) x_{i}(t),
\end{aligned}
$$

with initial state $x_{i}(0)=w(i)$ and spatial boundary condition $u_{0}(t)=d(t)$. Defining the semi-states $x_{t}(t, i):=x_{i}(t)$ and $x_{s}(t, i):=u_{i}(t)$ yields the following mixedcontinuous-discrete spatially-varying 2-D Roesser model (see [30]):

$$
\left[\begin{array}{c}
\dot{x}_{t}(t, i) \\
x_{s}(t, i+1)
\end{array}\right]=\left[\begin{array}{cc}
A(i) & B(i) \\
C(i) & 0
\end{array}\right]\left[\begin{array}{c}
x_{t}(t, i) \\
x_{s}(t, i)
\end{array}\right]
$$

with boundary conditions $x_{t}(0, \cdot)=w(\cdot)$ and $x_{s}(\cdot, 0)=d(\cdot)$. The evolution of the semi-states given boundary conditions $x_{t}(0,.) \in \ell_{2}$ and $x_{s}(., i) \in L_{2} \cap L_{\infty}$ is of particular interest in this paper.

Before proceeding, it is instructive to note that the aforementioned steady-state matching requirement considered in this paper simply translates to the requirement that $\lim _{s \rightarrow 0} G_{i}(s)=1$ for all $i \in \mathbb{Z}_{+}$. It is for this reason that sufficient conditions for string-stability which need the $H_{\infty}$ norm of each $G_{i}$ to be strictly less than unity (see e.g. [24]) do not apply directly. This is overcome via the analysis developed below. 


\section{String-Stability Analysis}

Consider the cascade shown in Figure 1 Let $G_{i}$ denote the rational transfer function from the input signal $u_{i}$ to the output signal $u_{i+1}$, at the LTI subsystem labelled $i \in \mathbb{Z}_{+}$, which has initial state $w(i)$.

Definition 1 The cascade in Figure 1 is called $L_{2}$-to- $L_{\infty}$ string-stable if there exists finite constants $M_{1}, M_{2}, M_{3}>0$ such that the bound

$$
\left\|u_{i}\right\|_{\infty} \leq M_{1}\|d\|_{2}+M_{2}\|d\|_{\infty}+M_{3}\|w\|_{2}
$$

holds for all $i \in \mathbb{Z}_{+}$and arbitrary boundary conditions $u_{0}=d \in L_{2} \cap L_{\infty}$ and $w=$ $\{w(0), w(1), \ldots\} \in \ell_{2}$.

The following theorem provides a sufficient condition for $L_{2}$-to- $L_{\infty}$ string-stability in terms of appropriate state-space realizations $(A(i), B(i), C(i))$ of the subsystem transfer functions $G_{i}$ and the corresponding 2-D Roesser model (10). This is used subsequently to establish another sufficient, but importantly decentralized, stringstability certificate for the cascade.

Theorem 1 Given $(A(i), B(i), C(i))$ for $i \in \mathbb{Z}_{+}$, consider the spatially-varying 2-D Roesser model (10). Suppose the following exist:

1. finite constants $\beta, \gamma, \lambda, \kappa>0$ such that, for all $i \in \mathbb{Z}_{+}$,

$$
\|B(i)\|_{\infty \rightarrow \infty} \leq \beta,\|C(i)\|_{\infty \rightarrow \infty} \leq \gamma \text {, and }\left\|e^{A(i) t}\right\|_{\infty \rightarrow \infty} \leq \kappa e^{-\lambda t} \forall t \in \mathbb{R}_{+} ;
$$

2. positive-semi-definite matrix sequences $\left\{P_{t}(0)=P_{t}(0)^{*}, P_{t}(1)=P_{t}(1)^{*}, \ldots\right\}$ and $\left\{P_{s}(0)=P_{s}(0)^{*}, P_{S}(1)=P_{s}(1)^{*}, \ldots\right\}$, and finite constants $\lambda_{s}, \lambda_{t}>0$ such that, for all $i \in \mathbb{Z}_{+}$,

(a) $\lambda_{\min }\left(P_{s}(i)\right) \geq \lambda_{s}, \lambda_{\max }\left(P_{t}(i)\right) \leq \lambda_{t}$ and

(b) $Q(i):=\tilde{A}(i)^{*} \tilde{P}_{t}(i)+\tilde{P}_{t}(i) \tilde{A}(i)+\tilde{A}(i)^{*} \tilde{P}_{s}(i+1) \tilde{A}(i)-\tilde{P}_{s}(i) \leq 0$,

where

$$
\tilde{A}(i):=\left[\begin{array}{cc}
A(i) & B(i) \\
C(i) & 0
\end{array}\right], \quad \tilde{P}_{t}(i):=\left[\begin{array}{cc}
P_{t}(i) & 0 \\
0 & 0
\end{array}\right] \quad \text { and } \quad \tilde{P}_{s}(i):=\left[\begin{array}{cc}
0 & 0 \\
0 & P_{s}(i)
\end{array}\right] .
$$

Then there exist finite constants $M_{1}, M_{2}, M_{3}>0$ such that

$$
|x(t, i)|_{\infty} \leq M_{1}\left\|x_{t}(0, \cdot)\right\|_{2}+M_{2}\left\|x_{S}(\cdot, 0)\right\|_{2}+M_{3}\left\|x_{s}(\cdot, 0)\right\|_{\infty}
$$

for all $(t, i) \in \mathbb{R}_{+} \times \mathbb{Z}_{+}$and arbitrary boundary conditions $x_{s}(\cdot, 0) \in L_{2} \cap L_{\infty}$ and $x_{t}(0, \cdot) \in \ell_{2}$.

Proof Let $V_{t}(t, i):=x_{t}(t, i)^{*} P_{t}(i) x_{t}(t, i)$ and $V_{s}(t, i):=x_{s}(t, i)^{*} P_{s}(i) x_{s}(t, i)$ for $(t, i) \in$ $\mathbb{R}_{+} \times \mathbb{Z}_{+}$. Using (10) and hypothesis 2(b), it follows that

$$
\begin{aligned}
\frac{\partial V_{t}}{\partial t}(t, i) & +V_{s}(t, i+1)-V_{s}(t, i) \\
& =x(t, i)^{*}\left(\tilde{A}(i)^{*} \tilde{P}_{t}(i)+\tilde{P}_{t}(i) \tilde{A}(i)+\tilde{A}(i)^{*} \tilde{P}_{s}(i+1) \tilde{A}(i)-\tilde{P}_{s}(i)\right) x(t, i) \\
& =x(t, i)^{*} Q(i) x(t, i) \leq 0 \quad \text { for all }(t, i) \in \mathbb{R}_{+} \times \mathbb{Z}_{+} .
\end{aligned}
$$


Since $V_{t}(t, i) \geq 0$ and $V_{s}(t, i) \geq 0$, the inequality (12) implies (2) holds by Lemma1 Combining this with the properties of $P_{s}(i)>0$ and $P_{t}(i) \geq 0$ identified in hypothesis 2(a) yields

$$
\begin{aligned}
\int_{0}^{t} x_{s}(\tau, i)^{*} x_{s}(\tau, i) d \tau & \leq \frac{1}{\lambda_{s}} \int_{0}^{t} V_{s}(\tau, i) d \tau \\
& \leq \frac{1}{\lambda_{s}} \int_{0}^{t} V_{s}(\tau, 0) d \tau+\frac{1}{\lambda_{s}} \sum_{k=0}^{i} V_{t}(0, k) \\
& =\frac{1}{\lambda_{s}} \int_{0}^{t} x_{s}(\tau, 0)^{*} P_{s}(0) x_{s}(\tau, 0) d \tau+\frac{1}{\lambda_{s}} \sum_{k=0}^{i} x_{t}(0, k)^{*} P_{t}(k) x_{t}(0, k) \\
& \leq \frac{\lambda_{\max }\left(P_{s}(0)\right)}{\lambda_{s}}\left\|x_{s}(\cdot, 0)\right\|_{2}^{2}+\frac{\lambda_{t}}{\lambda_{s}}\left\|x_{t}(0, \cdot)\right\|_{2}^{2} .
\end{aligned}
$$

Now bearing in mind hypothesis 1 , and noting that $x_{t}(0, \cdot) \in \ell_{2}$ implies $\left|x_{t}(0, i)\right|_{\infty} \leq$ $\left|x_{t}(0, i)\right|_{2} \leq\left\|x_{t}(0, \cdot)\right\|_{2}$ for all $i \in \mathbb{Z}_{+}$, it follows that

$$
\begin{aligned}
\left|x_{t}(t, i)\right|_{\infty} & =\left|x_{t}(0, i) e^{A(i) t}+\int_{0}^{t} e^{A(i)(t-\tau)} B(i) x_{s}(\tau, i) d \tau\right|_{\infty} \\
& \leq \kappa e^{-\lambda t}\left|x_{t}(0, i)\right|_{\infty}+\kappa \sup _{i}\|B(i)\|_{\infty \rightarrow \infty} \int_{0}^{t} e^{-\lambda(t-\tau)}\left|x_{s}(\tau, i)\right|_{\infty} d \tau \\
& \leq \kappa\left\|x_{t}(0, \cdot)\right\|_{2}+\kappa \beta\left(\int_{0}^{t}\left(e^{-\lambda(t-\tau)}\right)^{2} d \tau\right)^{\frac{1}{2}}\left(\int_{0}^{t}\left|x_{s}(\tau, i)\right|_{\infty}^{2} d \tau\right)^{\frac{1}{2}} \\
& \leq \kappa\left\|x_{t}(0, \cdot)\right\|_{2}+\kappa \beta\left(\frac{1-e^{-2 \lambda t}}{2 \lambda}\right)^{\frac{1}{2}}\left(\int_{0}^{t}\left|x_{s}(\tau, i)\right|_{2}^{2} d \tau\right)^{\frac{1}{2}} \\
& \leq \kappa\left\|x_{t}(0, \cdot)\right\|_{2}+\frac{\kappa \beta}{\sqrt{2 \lambda}} \sqrt{\int_{0}^{t} x_{s}^{*}(\tau, i) x_{s}(\tau, i) d \tau} \\
& \leq \kappa\left\|x_{t}(0, \cdot)\right\|_{2}+\frac{\kappa \beta}{\sqrt{2 \lambda}} \sqrt{\frac{\lambda_{\max }\left(P_{s}(0)\right)}{\lambda_{s}}\left\|x_{s}(\cdot, 0)\right\|_{2}^{2}+\frac{\lambda_{t}}{\lambda_{s}}\left\|x_{t}(0, \cdot)\right\|_{2}^{2}} \\
& \leq \kappa\left(1+\frac{\beta \sqrt{\lambda_{t}}}{\sqrt{2 \lambda \lambda_{s}}}\right)\left\|x_{t}(0, \cdot)\right\|_{2}+\frac{\kappa \beta \sqrt{\lambda_{\max }\left(P_{s}(0)\right)}}{\sqrt{2 \lambda \lambda_{s}}}\left\|x_{s}(\cdot, 0)\right\|_{2} \\
& :=\mu_{1}\left\|x_{t}(0, \cdot)\right\|_{2}+\mu_{2}\left\|x_{s}(\cdot, 0)\right\|_{2}
\end{aligned}
$$

for all $(t, i) \in \mathbb{R}_{+} \times \mathbb{Z}_{+}$. In particular, (14) holds by the Cauchy-Schwartz inequality, (15) holds because $\left|x_{s}(\tau, i)\right|_{\infty} \leq\left|x_{s}(\tau, i)\right|_{2}$ and (16) holds in view of (13).

Finally, note that $x_{s}(t, i+1)=C(i) x_{t}(t, i)$ by (10). Hence,

$$
\left|x_{S}(t, i+1)\right|_{\infty} \leq \sup _{i}\|C(i)\|_{\infty \rightarrow \infty}\left|x_{t}(t, i)\right|_{\infty} \leq \gamma\left(\mu_{1}\left\|x_{t}(0, \cdot)\right\|_{2}+\mu_{2}\left\|x_{S}(\cdot, 0)\right\|_{2}\right)
$$


for all $(t, i) \in \mathbb{R}_{+} \times \mathbb{Z}_{+}$, where (17) and hypothesis 1 have been used. Moreover, $x_{s}(\cdot, 0) \in L_{2} \cap L_{\infty}$ implies $\left|x_{s}(t, 0)\right|_{\infty} \leq\left\|x_{s}(\cdot, 0)\right\|_{\infty}$. As such, it follows that

$$
\begin{aligned}
|x(t, i)|_{\infty}= & \max \left\{\left|x_{t}(t, i)\right|_{\infty},\left|x_{s}(t, i)\right|_{\infty}\right\} \\
\leq & \max \left\{\mu_{1}\left\|x_{t}(0, \cdot)\right\|_{2}+\mu_{2}\left\|x_{s}(\cdot, 0)\right\|_{2},\right. \\
& \left.\quad \max \left\{\gamma\left(\mu_{1}\left\|x_{t}(0, \cdot)\right\|_{2}+\mu_{2}\left\|x_{s}(\cdot, 0)\right\|_{2}\right),\left\|x_{s}(\cdot, 0)\right\|_{\infty}\right\}\right\} \\
\leq & \max \{1, \gamma\} \mu_{1}\left\|x_{t}(0, \cdot)\right\|_{2}+\max \{1, \gamma\} \mu_{2}\left\|x_{s}(\cdot, 0)\right\|_{2}+\left\|x_{s}(\cdot, 0)\right\|_{\infty}
\end{aligned}
$$

for all $(t, i) \in \mathbb{R}_{+} \times \mathbb{Z}_{+}$, as claimed.

Theorem 1 and the following lemma lead to the main decentralized string-stability certificate summarised in Theorem 2 below.

Lemma 4 For all $i \in \mathbb{Z}_{+}$, suppose that $G_{i}(s):=C(i)(s I-A(i))^{-1} B(i)$ with $A(i) \in$ $\mathbb{R}^{n(i) \times n(i)}$ Hurwitz, and that $\left\|G_{i}\right\|_{\infty} \leq 1$. Then there exist positive-semi-definite matrix sequences $\left\{P_{t}(0)=P_{t}(0)^{*}, P_{t}(1)=P_{t}(1)^{*}, \ldots\right\}$ and $\left\{P_{S}(0)=P_{s}(0)^{*}, P_{s}(1)=P_{s}(1)^{*}, \ldots\right\}$ such that

$$
Q(i):=\tilde{A}(i)^{*} \tilde{P}_{t}(i)+\tilde{P}_{t}(i) \tilde{A}(i)+\tilde{A}(i)^{*} \tilde{P}_{s}(i+1) \tilde{A}(i)-\tilde{P}_{s}(i) \leq 0
$$

for all $i \in \mathbb{Z}_{+}$, where $\tilde{A}(i), \tilde{P}_{t}(i)$ and $\tilde{P}_{s}(i)$ are as defined in (11). Specifically, take $P_{s}(i)=I$ and $P_{t}(i)=P_{t}(i)^{*} \geq 0$ to be the unique solution of

$$
A(i)^{*} P_{t}(i)+P_{t}(i) A(i)+P_{t}(i) B(i) B(i)^{*} P_{t}(i)+C(i)^{*} C(i)=0
$$

that satisfies $\sigma\left(A(i)+B(i) B(i)^{*} P_{t}(i)\right) \in \mathbb{C}_{-} \cup j \mathbb{R}$.

Proof In view of Lemma 2, there exists a real matrix $X(i)=X^{*}(i) \geq 0$ such that

$$
X(i) A(i)+A(i)^{*} X(i)+X(i) B(i) B(i)^{*} X(i)+C(i)^{*} C(i)=0 .
$$

Applying the Schur complement to 20 gives

$$
\left[\begin{array}{cc}
X(i) A(i)+A(i)^{*} X(i)+C(i)^{*} C(i) X(i) B(i) \\
B(i)^{*} X(i) & -I
\end{array}\right] \leq 0 .
$$

Note that with $P_{t}(i)=X(i)$ and $P_{S}(i)=I$,

$$
Q(i)=\left[\begin{array}{cc}
X(i) A(i)+A(i)^{*} X(i)+C(i)^{*} C(i) X(i) B(i) \\
B(i)^{*} X(i) & -I
\end{array}\right],
$$

whereby (21) implies (18).

Theorem 2 Consider a cascade of stable LTI systems, as shown in Figure 1 Let $G_{i}(s)=C(i)(s I-A(i))^{-1} B(i)$, with $A(i) \in \mathbb{R}^{n(i) \times n(i)}$ Hurwitz, be a minimal balanced realization for subsystem $i \in \mathbb{Z}_{+}$and suppose that the following hold:

1. there exists a constant $\varsigma>0$ such that the minimum Hankel singular value of $G_{i}$ satisfies $\sigma_{n(i)} \geq \varsigma$ for all $i \in \mathbb{Z}_{+}$.

2. there exist finite constants $\beta, \gamma, \lambda, \kappa>0$ such that

$$
\|B(i)\|_{\infty \rightarrow \infty} \leq \beta,\|C(i)\|_{\infty \rightarrow \infty} \leq \gamma \text { and }\left\|e^{A(i) t}\right\|_{\infty \rightarrow \infty} \leq \kappa e^{-\lambda t} \text { for all }(t, i) \in \mathbb{R}_{+} \times \mathbb{Z}_{+} .
$$


If $\left\|G_{i}\right\|_{\infty} \leq 1$ for all $i \in \mathbb{Z}_{+}$, then the cascade is $L_{2}$-to- $L_{\infty}$ string-stable in the sense of Definition 1 .

Proof Let $\left\|G_{i}\right\|_{\infty} \leq 1$ for all $i \in \mathbb{Z}_{+}$, so that in view of Lemma2, Lemma3, Remark1 and hypothesis 1 above, the unique solution of $P_{t}(i) \geq 0$ of 19$)$ such that $\sigma(A(i)+$ $\left.B(i) B(i)^{*} P_{t}(i)\right) \in \mathbb{C}_{-} \cup j \mathbb{R}$ also satisfies $P_{t}(i)>0$ and $\lambda_{\max }\left(P_{t}(i)\right) \leq 1 / \varsigma$ for all $i \in \mathbb{Z}_{+}$. Using Lemma 4 and hypothesis 2 , it follows that Theorem 1 applies to yield the required bound on the semi-state evolution of the corresponding 2-D Roesser model (10), given boundary conditions $x_{s}(\cdot, 0)=d \in L_{2} \cap L_{\infty}$ and $x_{t}(0, \cdot)=w \in \ell_{2}$. As such, the result holds since $u_{i}(t)=x_{s}(t, i)$.

\section{Distant-Downstream Control of Irrigation Channels}

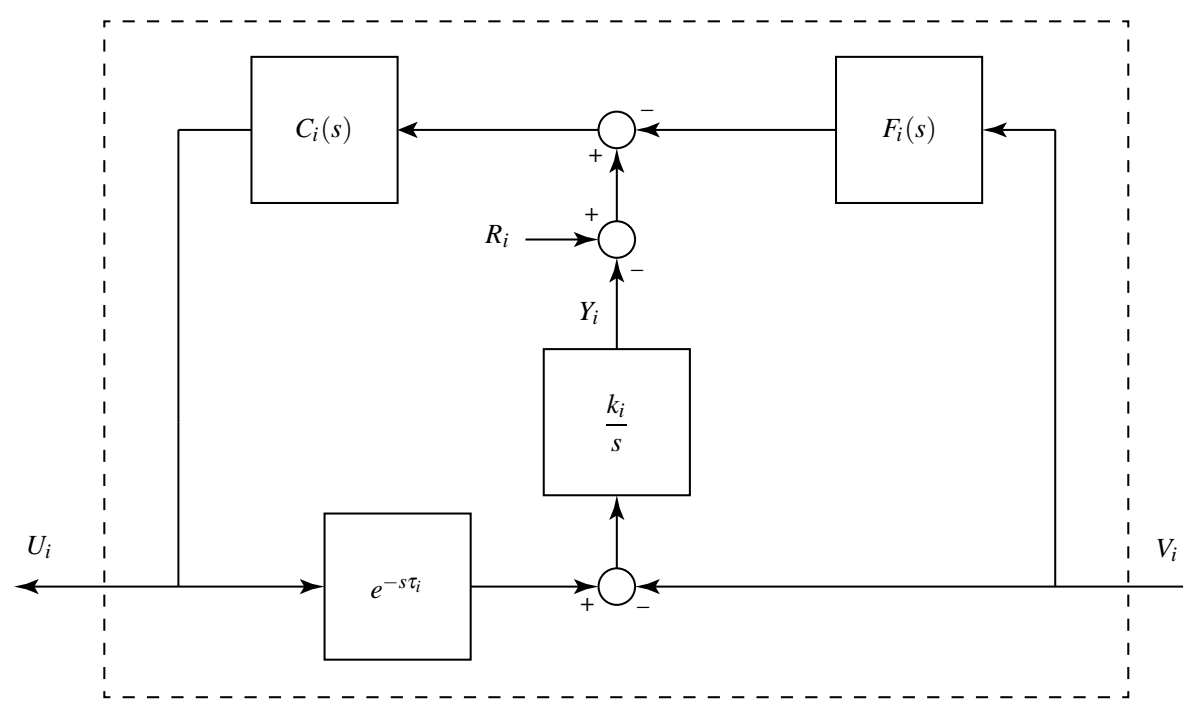

Fig. 2 Section (pool) of an irrigation channel under decentralized distant-downstream control.

Irrigation channels are used around the world to distribute fresh water for agriculture. The channels are typically sectioned into pools that stretch between gates that can be adjusted to locally impose gravity-powered flow downstream and at supply points. Figure 2 shows the block diagram for a section of an automated irrigation channel that has a so-called distant-downstream control architecture $[4,22,29,20,17$, 19,25]. The local feedback controller $C_{i}(s)$ is used to regulate the water-level $y_{i}$ at the downstream gate, which reflects the capacity of the section to supply flow locally and downstream, under the power of gravity. This is achieved via adjustment of the inflow $u_{i}$ at the upstream gate, in response to variation of the outflow load $v_{i}$. The controlled inflow $u_{i}$ is a load on the upstream section. A correspondingly automated irrigation channel is therefore a cascade of subsystems on the kind shown in Figure 2 Importantly, the distant-downstream control architecture translates to demand-driven 
Table 1 Pool model and controller parameters, $n=0,1,2, \cdots$.

\begin{tabular}{c|cc|ccc}
\hline \hline \multirow{2}{*}{ Pool number $(i)$} & \multicolumn{2}{|c|}{ Pool Model Parameters } & \multicolumn{3}{c}{ Controller Parameters } \\
\cline { 2 - 6 } & time-delay $\left(\tau_{i}\right)$ & $k_{i}$ & $K_{i}$ & $p_{i}$ & $z_{i}$ \\
\hline $5 n+1$ & 8 & $1 / 22414$ & 101.25 & $1 / 8.7$ & $1 / 77$ \\
\hline $5 n+2$ & 3 & $1 / 11942$ & 329.74 & $1 / 350$ & $1 / 30$ \\
\hline $5 n+3,5 n+4,5 n+5$ & 16 & $1 / 43806$ & 65 & $1 / 15.2$ & $1 / 128$ \\
\hline
\end{tabular}

release of water from upstream storage, which has merit from an operations perspective in light of the limited availability of storage in the channels.

In this section, a heterogeneous channel of pools with specifications as in Table 1 are considered, where the pool delay and integrator constant are denoted by $\tau_{i}$ and $k_{i}$, respectively, and PI controller is represented by $C_{i}(s)=K_{i} \frac{\left(s+z_{i}\right)}{s\left(s+p_{i}\right)}$ for each pool $i$.

The conventional decentralized distant-downstream control architecture corresponds to $F_{i}(s)=0$ in Figure 2. For a homogeneous channel and integral action in identical decentralized controllers $C(s)$, transient flow peaks produced along the channel in response to a step increase in downstream flow, are amplified as these propagate upstream [18,2]. Indeed, it is not possible to achieve $\|G\|_{\infty} \leq 1$, where $G=\frac{C(s) k / s}{1+C(s) k e^{-s \tau} / s}$ is the transfer function from outflow $v$ to inflow $u$. Even in the case of heterogeneous channels, under a purely decentralized distant-downstream control architecture transient water flow peaks can be amplified, as shown in Figure 3 for the channel and controller data summarised in Table 1 Note that in this case, $\left\|G_{1}\right\|_{\infty}=1.66,\left\|G_{2}\right\|_{\infty}=2.16,\left\|G_{3}\right\|_{\infty}=\left\|G_{4}\right\|_{\infty}=\left\|G_{5}\right\|_{\infty}=1.836$, and so on.

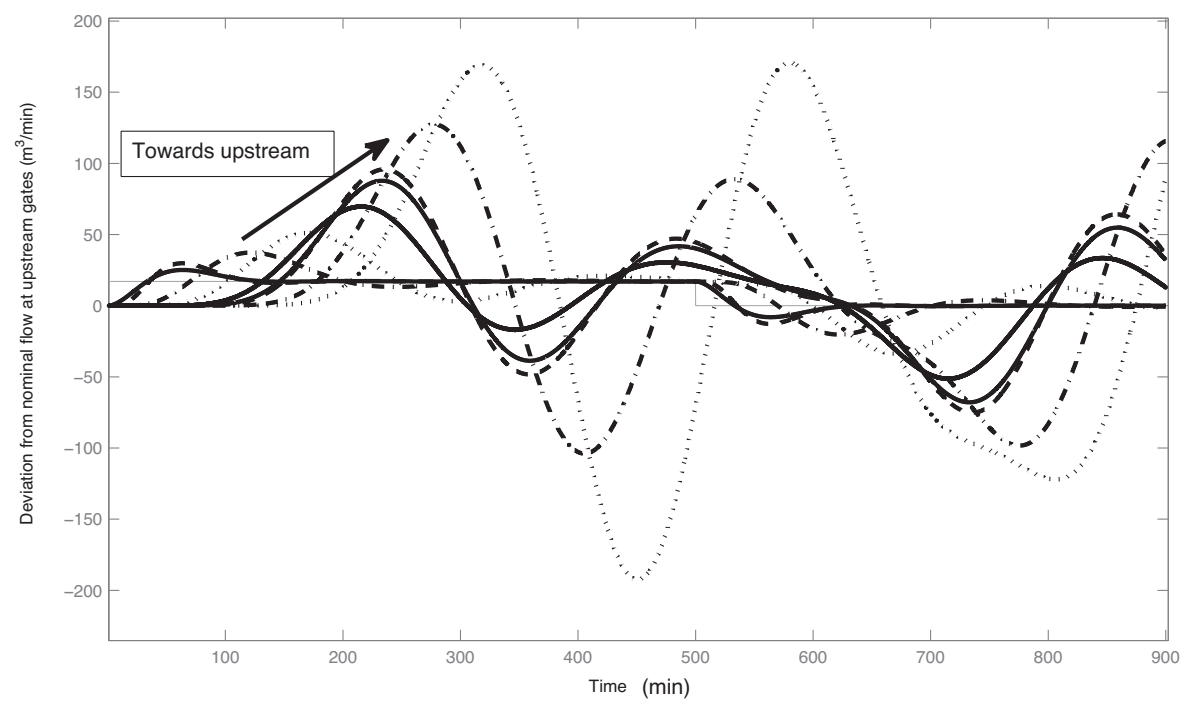

Fig. 3 Response of channel under decentralized distant-downstream control - i.e., $F_{i}(s)=0$ and $C_{i}(s)=$ $K_{i} \frac{\left(s+z_{i}\right)}{s\left(s+p_{i}\right)}$. 
As first considered in [26, 25], the local feed-forward controller $F_{i}(s)$ provides scope for shaping the transfer function $G_{i}(s)$ from $v_{i}$ to $u_{i}$ for each section of the automated channel, as desired. Of course, there is a price to pay; the steady-state offset in water-level relative to a constant reference for a step increase in flow load becomes non-zero. For a given PI controller $C_{i}(s)$ (i.e., given $K_{i}, z_{i}, p_{i}>0$ ), a desired stable strictly-proper transfer function $G_{i}(s)$, which must satisfy $G_{i}(0)=1$ to ensure steady-state matching of inflow to outflow, can be achieved by setting

$$
F_{i}(s)=\frac{k_{i}}{s}\left(1-G_{i}(s) e^{-s \tau_{i}}\right)-\frac{G_{i}(s)}{C_{i}(s)} .
$$

Note that $F_{i}(s)$ is stable, because $G_{i}(s)$ is stable, $\left(1-G_{i}(s) e^{-s \tau_{i}}\right)$ has at least one zero at $s=0$ and the zero of $C_{i}(s)$ has negative real part. Note that it is also reasonable to use a rational approximation of the delay transfer function $e^{-s \tau_{i}}$, provided the error is sufficiently small up to the loop-gain crossover frequency, as the closed-loop behaviour is insensitive to such modeling uncertainty. For example, the Padé approximation $\left(1-s \tau_{i} / 2\right) /\left(1+s \tau_{i} / 2\right)$ is acceptable provided the controller gain $K_{i}$ and corresponding loop-gain crossover frequency are sufficiently small, which is necessary to achieve reasonable control performance and robustness anyway [17].

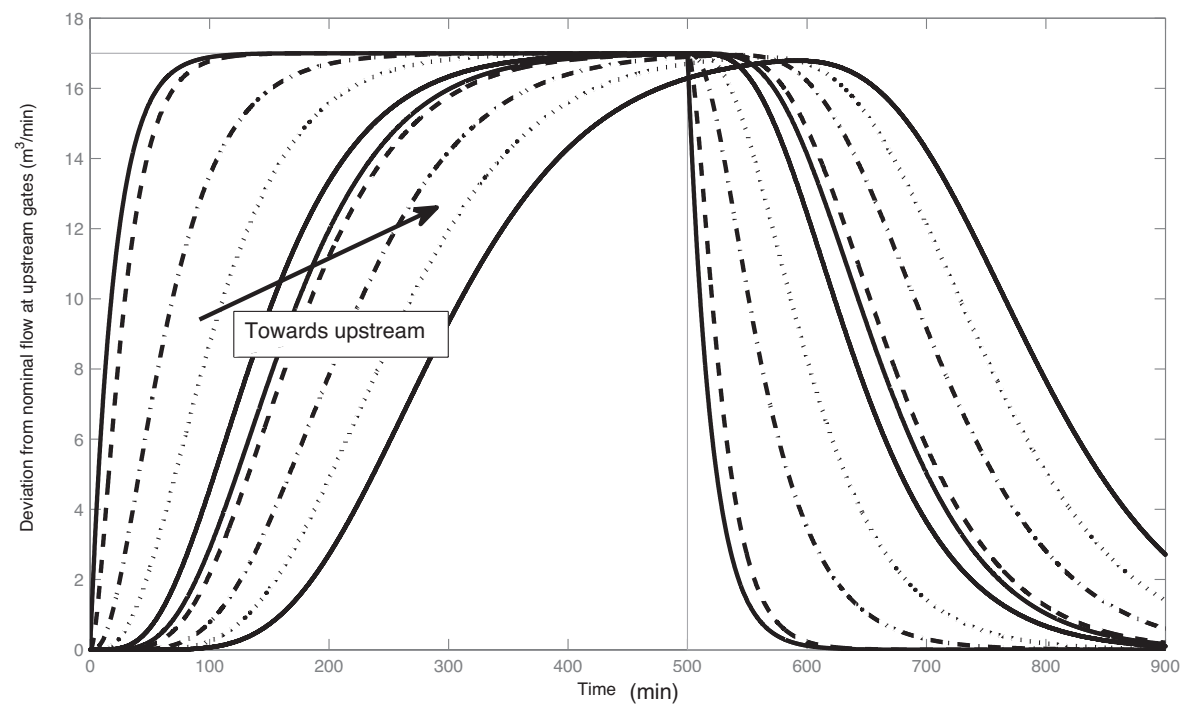

Fig. 4 Response of channel under decentralized distant-downstream control with $F_{i}(s)$ set as in [22] so that $G_{1}(s)=1 /(20 s+1), G_{2}(s)=1 /(10 s+1), G_{3}(s)=G_{4}(s)=G_{5}(s)=1 /(40 s+1), \cdots$.

By Theorem 2, choosing $F_{i}(s)$ to achieve $\left\|G_{i}\right\|_{\infty} \leq 1$ for each pool would imply $L_{2}$-to- $L_{\infty}$ string-stability of the automated channel. That is, uniformly bounded flow peaks in response to finite-duration step-changes of flow load. One possible choice is $G_{i}(s)=1 /\left(T_{i} s+1\right)$ for some time-constant $T_{i}>0$; note $G_{i}(0)=1$. In this case, $\left\|g_{i}\right\|_{1}:=\int_{0}^{\infty}\left|g_{i}(t)\right| d t \leq 1$, where $g_{i}(\cdot)$ denotes the impulse response associated with 
the transfer function $G_{i}$ (i.e. $g_{i}(t)=e^{-t / T_{i}}$ here). In fact, $\left\|g_{i}\right\|_{1} \leq 1$, which implies $\left\|G_{i}\right\|_{\infty} \leq 1$ (in general), is a condition that ensures non-amplification of peaks as these propagate; see [26]. This is illustrated in Figure 4, where again a $17 \mathrm{~m}^{3} / \mathrm{min}$ step change in the outflow of the bottom pool is considered.

With $F_{i}(s)$ selected to achieve $G_{1}(s)=0.03 /\left(s^{2}+0.28 s+0.03\right), G_{2}(s)=0.06 /\left(s^{2}\right.$ $+0.35 s+0.06)$, and $G_{3}(s)=G_{4}(s)=G_{5}(s)=0.01 /\left(s^{2}+0.15 s+0.01\right)$, one has $G_{i}(0)=1$ and $\left\|G_{i}\right\|_{\infty}=1$ for all $i$. Thus, $L_{2}$-to- $L_{\infty}$ stability would be achieved by Theorem 2. The controlled flow responses to a $17 \mathrm{~m}^{3} / \mathrm{min}$ step change in the outflow at the bottom pool are shown in Figures 5 and 6 It can be seen that, while there is amplification of flow peaks as these propagate along the bottom pools, this does not persist and the peak flows remain uniformly bounded along the channel as expected.

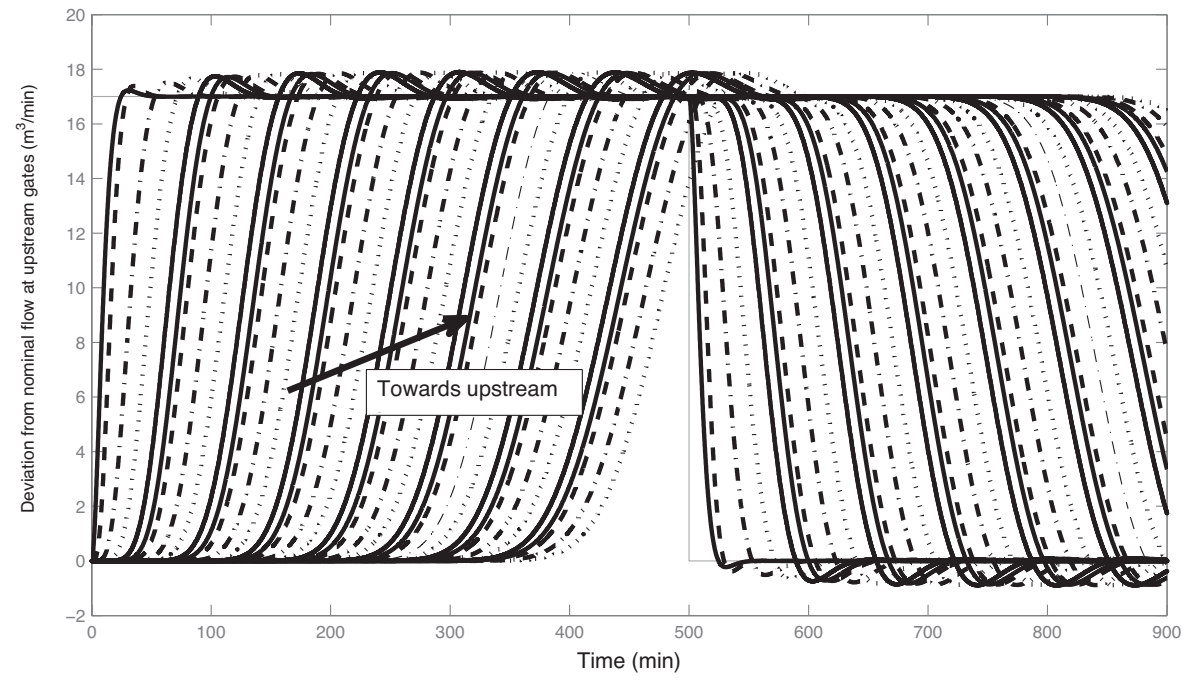

Fig. 5 Response of channel under decentralized distant-downstream control with $F_{i}(s)$ set as in 22 so that $\left\|G_{i}\right\|_{\infty}=1$.

\section{Conclusion}

An $L_{2}$-to- $L_{\infty}$ string-stability property is defined and analyzed for a heterogeneous cascade of stable LTI subsystems, subject to the requirement that the interconnection signals match the constant spatial boundary conditions in steady state. A sufficient condition is established in terms of state-space realizations for the subsystems. This is subsequently used to develop a decentralized string-stability certificate, which simply involves a location independent bound on the $H_{\infty}$ norm of the transfer function relating the interconnection signals associated with each subsystem. An application of this is explored within the context of scalable distant-downstream controller design in irrigation channels. It would be of interest to understand if the $H_{\infty}$ norm condition 


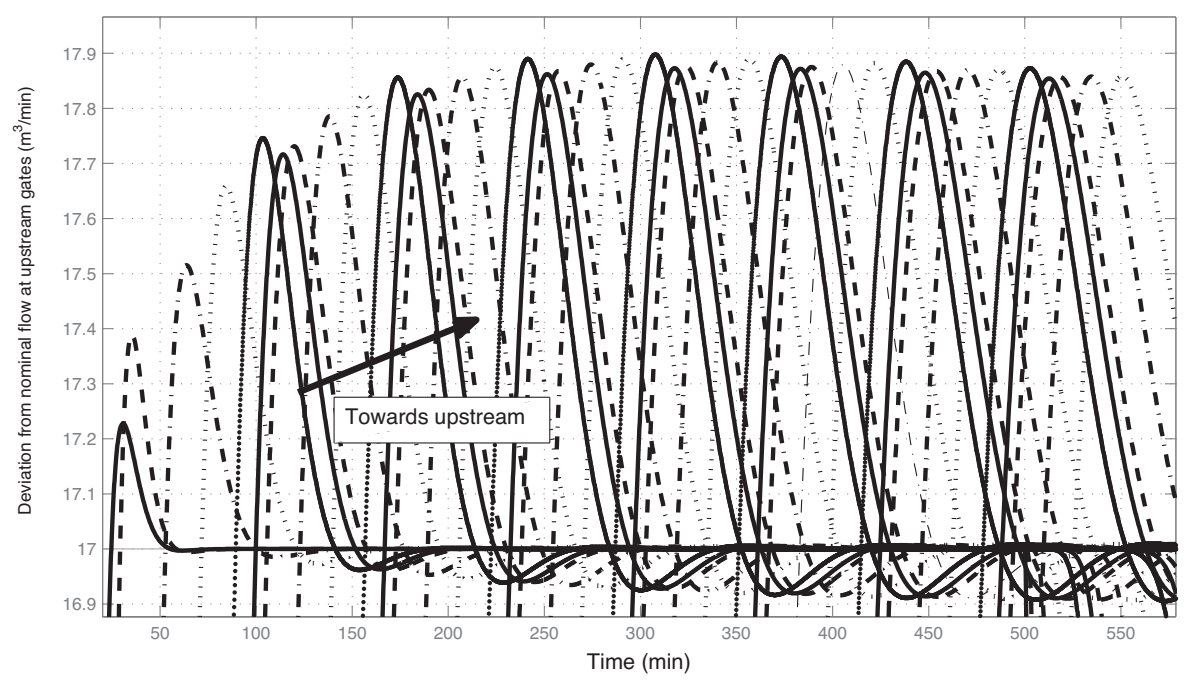

Fig. 6 Response channel under decentralized distant-downstream control with $F_{i}(s)$ set as in 22 so that $\left\|G_{i}\right\|_{\infty}=1$ (zoomed in).

is necessary for $L_{2}$-to- $L_{\infty}$ stability of homogeneous cascades. The robustness properties of the presented local feed-forward approach to satisfying the decentralized string-stability certificate within the context of irrigation channel control-system design also requires further investigation.

\section{References}

1. Arneson, H., Langbort, C.: A linear programming approach to routing control in networks of linear positive systems. Automatica 48(5) (2012)

2. Cantoni, M., Weyer, E., Li, Y., Ooi, S., Mareels, I., Ryan, M.: Control of large-scale irrigation networks. Proceedings of the IEEE 95, 75-91 (2007)

3. Chu, K.: Decentralized control of high-speed vehicular strings. Transportation Science 8, $361-384$ (1974)

4. Clemmens, A., Replogle, J.: Control of irrigation canal networks. Journal of Irrigation and Drainage Engineering 115(1), 96-110 (1989)

5. Dabkowski, P., Galkowski, K., Bachelier, O., Rogers, E.: Control of discrete linear repetitive processes using strong practical stability and $H_{\infty}$ disturbance attenuation. Systems and Control Letters $61,1138-$ $1144(2012)$

6. Darbha, S., Hedrick, J.K.: String stability of interconnected systems. IEEE Transactions on Automatic Control 41(3), 349-357 (1996)

7. Disney, S., Towilla, D., Velde, W.: Variance amplification and the golden ratio in production and inventory control. International Journal of Production Economics 90, 259-309 (2004)

8. Du, C., Xie, L.: $H_{\infty}$ Control and Filtering of Two-Dimensional Systems. Springer-Verlag (2002)

9. Eyre, J., Yanakiec, D., Kanellakopoulos, I.: A simplified framework for string stability analysis of automated vehicles. Vehicle System Dynamics 30, 375-405 (1998)

10. Huang, X.Y., Yan, N., Guo, H.: An $H_{\infty}$ control method of the bullwhip effect for a class of supply chain system. International Journal of Production Research 45, 207-226 (2007)

11. Ioannou, P.A., Chien, C.C.: Autonomous intelligent cruise control. IEEE Transactions on Vehicular Technology 42(4), 657-672 (1993) 
12. Klinge, S., Middleton, R.H.: String stability analysis of homogeneous linear unidirectionally connected systems with nonzero initial conditions. Proceedings of Joint 48th IEEE Conference on Decision and Control and 28th Chinese Control Conference pp. 17-22 (2009)

13. Knorn, S.: A two-dimensional systems stability analysis of vehicle platoons. Ph.D. thesis, National University of Ireland (2013)

14. Knorn, S., Middleton, R.H.: Stability of two-dimensional linear systems with singularities on the stability boundary using LMIs. IEEE Transactions on Automatic Control 58, 2579 - 2590 (2013)

15. Lestas, I., Vinnicombe, G.: Scalable decentralized robust stability certificates for networks of interconnected heterogeneous dynamical systems. IEEE Transactions on Automatic Control 51(10), 16131625 (2006)

16. Levine, W., Athans, M.: On the optimal error regulation of a string of moving vehicles. IEEE Transactions on Automatic Control 11, 355 - 361 (1966)

17. Li, Y.: Robust control of open water channels. Ph.D. thesis, University of Melbourne, Department of Electrical and Electronic Engineering (2006)

18. Li, Y., Cantoni, M., Weyer, E.: On water-level error propagation in controlled irrigation channels. In: Proceedings Joint 44th IEEE Conference on Decision and Control and European Control Conference, pp. 2101-2106 (2005)

19. Litrico, X., Fromion, V.: Modeling and control of hydrosystems. Springer (2009)

20. Mareels, I., Weyer, E., Ooi, S.K., Cantoni, M., Li, Y., Nair, G.: System engineering for irrigation: Successes and challenges. Annual Reviews in Control 29(2), 191-204 (2005)

21. Peppard, L.: String stability of relative-motion PID vehicle control systems. Automatic Control, IEEE Transactions on 19(5), 579-581 (1974)

22. Schuurmans, J., Hof, A., Dijkstra, S., Bosgra, O., Brouwer, R.: Simple water level controller for irrigation and drainage canals. Journal of irrigation and drainage engineering 125(4), 189-195 (1999)

23. Seiler, P., Pant, A., Hedrick, K.: Disturbance propagation in vehicle strings. IEEE Transactions on Automatic Control 49(10), 1835-1841 (2004)

24. Shaw, E., Hedrick, J.K.: String stability analysis for heterogenous vehicle strings. In: Proceedings of American Control Conference, pp. 3118 - 3125 (2007)

25. Soltanian, L.: Distributed distant-downstream controller design for large-scale irrigation channels. Ph.D. thesis, University of Melbourne (2014)

26. Soltanian, L., Cantoni, M.: Achieving string stability in irrigation channels under distributed distantdownstream control. In: Proceedings of the IEEE Conference on Decision and Control (2013)

27. Wang, B., Zhang, F.: Some inequalities for the eigenvalues of the product of positive semidefinite hermitian matrices. Linear algebra and its applications 160, 113-118 (1992)

28. Weitz, L.A.: Investigating string stability of a time-history control law for interval management. Transportation Research Part C: Emerging Technologies 33, 257-271 (2011)

29. Weyer, E.: Decentralised PI control of an open water channel. In: Proceedings of the 15th IFAC world congress (2002)

30. Xiao, Y.: Stability test for 2-D continuous-discrete systems. In: Proceedings of the IEEE Conference on Decision and Control, pp. 3649-3654 (2001)

31. Zhou, K., Doyle, J.C., Glover, K.: Robust and optimal control. Prentice Hall (1996) 


\section{Author Biography: LAVEN SOLTANIAN}

Laven Soltanian was born in Tehran, Iran. She received the B.Sc. degree in Electrical Engineering from Iran University of Science and Technology in 2006, and the M.Sc. degree in Electrical Engineering, with a major in Control Systems, from K.N. Toosi University of Technology in 2009. She completed a Ph.D. degree in 2014 with the Department of Electrical and Electronic Engineering at the University of Melbourne. She then joined Rubicon Water, Australia, where she now works as a control engineer. Her research interests include automation and string-stability analysis of large scale systems such as irrigation networks, multi-dimensional systems, and robust control. 


\section{Author Biography: MICHAEL CANTONI}

Michael Cantoni was born in Australia. He received the B.Sc. degree in applied mathematics and the B.E. degree in electrical engineering (with honors) from the University of Western Australia, Crawley, in 1995, and the Ph.D. degree from the University of Cambridge, U.K., in 1998.

From 1998 to 2000, he held postdoctoral positions with the Department of Engineering at the University of Cambridge, and St. Johns College, Cambridge. Since then he has been with the University of Melbourne, Australia, where he currently holds the position of full Professor in the Department of Electrical and Electronic Engineering. He has served as a member of the editorial boards of IET Control Theory and Applications (2007-2010) and Systems \& Control Letters (2007-2014). In 2014, he was co-recipient of the IEEE CSS Control Systems Technology Award. His current research interests include robust and optimal control theory, distributed control and optimization of networks, model ap-

proximation, multidimensional systems, and related applications such as the automation of large-scale water distribution networks. 


\section{University Library}

\section{- M M N E R VA A gateway to Melbourne's research publications}

Minerva Access is the Institutional Repository of The University of Melbourne

Author/s:

Soltanian, L;Cantoni, M

Title:

Decentralized string-stability analysis for heterogeneous cascades subject to load-matching requirements

Date:

2015-10-01

Citation:

Soltanian, L. \& Cantoni, M. (2015). Decentralized string-stability analysis for heterogeneous cascades subject to load-matching requirements. MULTIDIMENSIONAL SYSTEMS AND SIGNAL PROCESSING, 26 (4), pp.985-999. https://doi.org/10.1007/s11045-015-0335-6.

Persistent Link:

http://hdl.handle.net/11343/283167 